\title{
Controlled Synthesis of Hyperbranched Polymers by TEMPO-Mediated Radical Polymerization
}

\author{
Mustafa Ciftci ${ }^{1,2}$ \\ ${ }^{1}$ Department of Chemistry, Faculty of Engineering and Natural Science, \\ Bursa Technical University, Bursa 16310, Turkey \\ ${ }^{2}$ Department of Chemistry, Faculty of Science and Letters, \\ Istanbul Technical University, 34469 Maslak, Istanbul, Turkey \\ mustafaciftcis@gmail.com
}

\begin{abstract}
Branched polymers with tunable branching densities are prepared by TEMPO-mediated radical polymerization. Thus, styrene is copolymerized with 4-methacryloyloxy-2,2,6,6tetramethylpiperidine-1-oxyl (TEMPO methacrylate) by using 2,2'-azobis(2methylpropionitrile) (AIBN) as initiator at $125^{\circ} \mathrm{C}$. The method is based on the use of TEMPO methacrylate as branching unit that possessing a nitroxyl stable radical moiety as propagation radical trap and a polymerizable methacrylate group. Depending on the concentration of TEMPO methacrylate and polymerization time, hyperbranched polymers with different branching densities are obtained.
\end{abstract}

Keywords: Hyperbranched polymers, NMRP, Controlled/living polymerization

\section{Introduction}

Hyperbranched polymers (HBPs), a unique class of dendritic macromolecules, have gained growing interest based on their exceptional characteristics including reduced solution viscosity, increased level of terminal functionality, and high solubility values $[1,2]$. Standing on these unique properties, they have been widely employed in numerous applications such as drug delivery systems, bioimaging agents, gene carriers, viscosity modifiers, and catalysis [3-5]. Furthermore, HBPs can be synthesized by comparatively more straightforward synthetic approaches compared to the other dendritic macromolecules. So far, three main methods have been introduced for their formation; (i) step growth polycondensation of $\mathrm{ABx}$ monomers, (ii) ring-opening multibranching polymerization and (iii) self-condensing vinyl polymerization (SCVP) [6-8]. The latter method, SCVP, seems to be arguably the most utilized technique as a result of its operational simplicity and lower risk of gelation [9-11]. The approach is based on the polymerization of initiator monomers (inimers), which possesses both polymerizable vinyl group and a pendant moiety capable of initiating the polymerization. Recently, we have presented the possibility of the combination of SCVP and light induced processes for the synthesis of HBPs. By precise selection of the inimer, either light-triggered halide abstraction [12] or hydrogen abstraction by Type II photoinitiators [13-15] was utilized for the in situ preparation of HBPs. The application of the formed polymers as useful materials in hydrophobic coatings, drug delivery and optical imaging was also illustrated [16-19].

Recently, a great deal of focus has been concentrated on the controlled/living radical polymerization (C/LRP) strategies because of their capability to yield well-defined polymers with narrow-molecular-weight distributions and beneficial end-functionalities [20,21]. The most important examples of such systems are nitroxidemediated radical polymerization (NMRP) [22], atom transfer radical polymerization (ATRP) [23] and reversible addition-fragmentation chain transfer polymerization (RAFT) [24]. NMRP, together with ATRP and RAFT, has proven to be a robust tool for synthesis of various macromolecular architectures for instance block and graft copolymers $[25,26]$. In this process, the control is accomplished through the reversible deactivation of growing radicals by stable radical such as $2,2,6,6$ - 
tetramethyl-1-piperidinyloxy (TEMPO).

Over the years, SCVP has been effectively practiced also in the controlled polymerization systems including NMRP [27-29], ATRP [30] and RAFT [31] by variation of the inimer structure. For instance, Yagci and coworkers recently reported a stylish approach for the syntheses of hyperbranched under mild conditions by the synergic combination of SCVP and recently developed metal-free ATRP [32]. In the process, tertiary and benzylic bromide functional inimers (either 2-(2bromoisobutryloxy)ethyl methacrylate or 4vinylbenzyl bromide were copolymerized with methyl methacrylate and styrene (St), respectively by using perylene as photocatalyst.

In the current study, hyperbranched polymers were synthesized via TEMPO-mediated copolymerization of styrene with TEMPO methacrylate. In the process, TEMPO methacrylate plays a crucial role as branching agent as it possesses both a polymerizable methacrylate group and stable nitroxyl radical.

\section{Experimental}

Styrene (St; 99\%, Aldrich) was passed through a basic alumina column to remove the inhibitor prior to use. TEMPO methacrylate (98\%, Aldrich), dimethylformamide (DMF, 99.8\%, Aldrich), tetrahydrofuran (THF, $\geq 99 \%$, Merck) and methanol $(99.9 \%$, Aldrich) were used as received.

${ }^{1} \mathrm{H}$ NMR spectra were recorded on a Bruker Advance III, $300 \mathrm{MHz}$ spectrometer at room temperature in $\mathrm{CDCl}_{3}$ with tetramethylsilane as an internal standard. Molecular weights and distributions were determined by GPC using Viscotek GPC max system including a pump module (GPC max, Viscotek, Houston, TX) and a refractive index (RI) detector (VE 3580, Viscotek). In analyses, $1 \mathrm{~mL} / \mathrm{min}$ flow rate and $50 \mu \mathrm{L}$ injection volume were used with autosampler system. The calibration of RI detector was done by narrow molecular weight polystyrene standards. Two columns (LT5000L, Mixed, Medium Organic $300 \times$ $8 \mathrm{~mm}$ and LT3000L, Mixed, Ultra-Low Organic 300 $\times 8 \mathrm{~mm}$ ) with a guard column (TGuard, Organic Guard Column $10 \times 4.6 \mathrm{~mm}$ ) were used for the tetrahydrofuran eluent at $35^{\circ} \mathrm{C}$. Viscotek OmniSEC Omni01 software was used to analyze the data.

A representative copolymerization procedure was as follows. St $(1 \mathrm{~mL}, 8.69 \mathrm{mmol})$, TEMPO methacrylate $(5 \mathrm{~mol} \%)$ and toluene $(1 \mathrm{~mL})$ were placed in a Schlenk tube equipped with a magnetic stirring bar and degassed by three freeze-pump- thaw cycles and left in a vacuum. Then, the tube was placed into an oil bath at $125^{\circ} \mathrm{C}$. After given time, the obtained polymers were precipitated in excess methanol and then dried under reduced pressure. Monomer conversion was determined gravimetrically.

A representative chain extension polymerization procedure was as follows. $1 \mathrm{~mL}$ of styrene were added to a homogeneous solution of above obtained hyperbranched polymer $(100 \mathrm{mg})$ in $1 \mathrm{~mL}$ of toluene in a Schlenk tube equipped with a magnetic stirring bar and degassed by three freeze-pumpthaw cycles and left in a vacuum. Then, the tube was placed into an oil bath at $125^{\circ} \mathrm{C}$. After given time, the obtained polymers were precipitated in excess methanol and then dried under reduced pressure. Monomer conversion was determined gravimetrically.

\section{Results and discussion}

Described method involves the radical polymerization of styrene by using AIBN as initiator at $125{ }^{\circ} \mathrm{C}$ in the presence of TEMPO methacrylate. While styrene takes part only in the formation of linear polymer chains, TEMPO methacrylate provides branching the generation of branching points by trapping the propagating radical. The overall process is illustrated in Scheme 1.

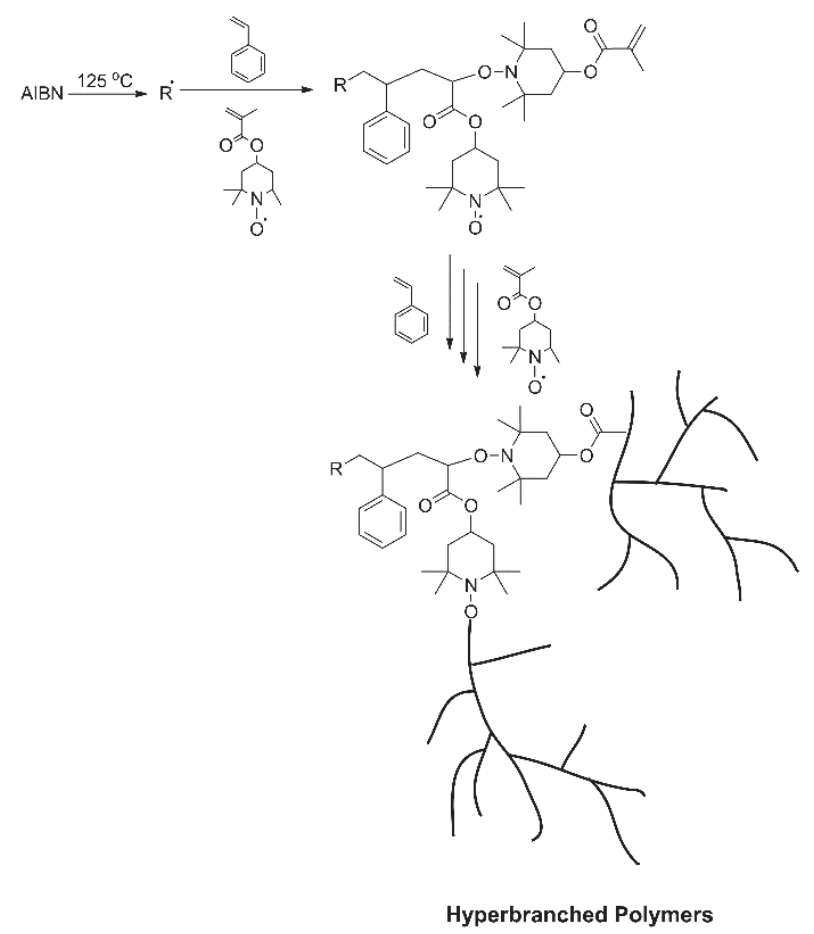

Scheme 1. Branching mechanism in TEMPO-mediated radical copolymerization of St and TEMPO methacrylate. 
Table 1. Synthesis of hyperbranched polymers by TEMPO-mediated radical polymerization.

\begin{tabular}{lccccc}
\hline Sample & $\begin{array}{c}\text { [St]/[TEMPO-methacrylate]/[AIBN] } \\
(\mathbf{m o l} \text { \%) }\end{array}$ & $\begin{array}{c}\text { Time } \\
\mathbf{( h )}\end{array}$ & $\begin{array}{c}\boldsymbol{M}_{\mathbf{n}, \mathbf{G P C}-\mathbf{R I}}{ }^{\mathbf{a}} \\
\left.\text { [g mol }^{-1}\right]\end{array}$ & $\begin{array}{c}\boldsymbol{M}_{\mathbf{n}, \mathbf{G P C}-\mathbf{L S}}^{\mathbf{b}} \\
{\left[\mathbf{g ~ m o l}^{-1}\right]}\end{array}$ & $\boldsymbol{R}_{\mathbf{M}} \mathbf{c}^{\mathbf{c}}$ \\
\hline HBPS-1 & $100 / 2.5 / 2.5$ & 24 & 5200 & 12700 & 0.41 \\
HBPS-2 & $100 / 5 / 5$ & 24 & 3500 & 9800 & 0.36 \\
HBPS-3 & $100 / 7.5 / 7.5$ & 24 & 2300 & 6800 & 0.31 \\
HBPS-4 & $100 / 5 / 5$ & 12 & 2250 & 6700 & 0.34 \\
HBPS-5 & $100 / 5 / 5$ & 36 & 3800 & 11900 & 0.32 \\
\hline
\end{tabular}

${ }^{\mathrm{a}}$ With refractive index detector. ${ }^{b}$ With light scattering detector. ${ }^{\mathrm{c}} R_{\mathrm{M}}=M_{\mathrm{n}, \mathrm{GPC}-\mathrm{RI}} / M_{\mathrm{n}, \mathrm{GPC}-\mathrm{LS}}$.

The typical results of the polymerization performed at different experimental paradigms are listed in Table 1. As can be deduced, formulations containing higher feed ratio of TEMPO methacrylate and initiator produced polymer with lower molecular weights. Moreover, the effect of TEMPO methacrylate concentration on the branching densities was also investigated via GPC measurements; as reported in literature the ratio between $M_{\mathrm{n}, \mathrm{GPC}-\mathrm{RI}}$ and $M_{\mathrm{n}, \mathrm{GPC}-\mathrm{LS}}\left(R_{\mathrm{M}}=M_{\mathrm{n}, \mathrm{GPC}-\mathrm{RI}} /\right.$ $M_{\mathrm{n}, \mathrm{GPC}-\mathrm{LS})}$ gave qualitative knowledge about the branching density of the polymers considering the more compact structures of the branched polymers than then linear polymers for a specific molecular weight. As can be seen from the Table, more branched polymers were obtained as the content of TEMPO methacrylate is increased. Similar behavior has been recorded for numerous traditional SCVP systems [12] using inimers rather than stable radical containing monomer, TEMPO methacrylate, which was employed in the current study.

The effect of polymerization time on the molecular weight and branching densities was also examined (Table 1, HBPS- 2, 4 and 5). As expected, higher polymerization times resulted in higher molecular weights and branching densities.

Moreover, the structure of the resulting hyperbranched polymers were confirmed by ${ }^{1} \mathrm{H}$ NMR analyses. As can be seen from Fig. 1, the methyl proton of the TEMPO ring ( $\sim$ снос̈m) was clearly detectable around $3.8 \mathrm{ppm}$ in addition to the aromatic polystyrene signals.

As a result of the mechanism, chains are terminated with TEMPO moiety that remain in the final branched polymer which enables the utilizing

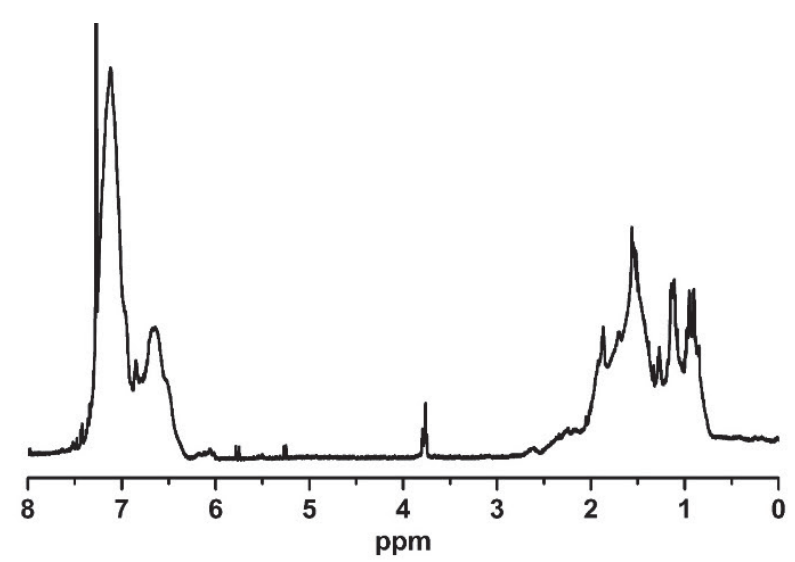

Fig. 1. ${ }^{1} \mathrm{H}$ NMR spectrum of hyperbranched polymer HBFS-2.

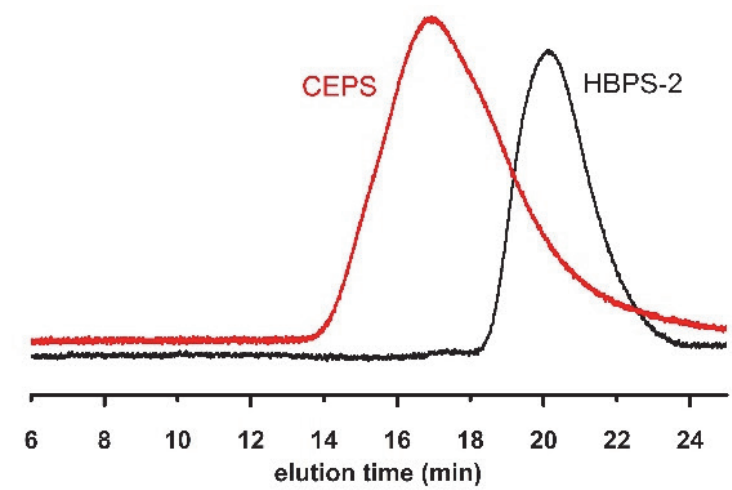

Fig. 2. SEC traces for the successful chain extension (HBPS-2 and CEPS).

of the obtained polymers as macroinitiator for further polymerization. As temperature increases, the branched polymer cleaves into stable (TEMPO) and initiating carbon-based radicals. Accordingly, the obtained branched polymer (HBPS-2, in Fig. 2) was used as macroinitiator in the chain extension 
polymerization of styrene in absence of any external radical initiator addition at $125^{\circ} \mathrm{C}$. After given time the product (CEPS, in Fig. 2) was precipitated, purified, characterized and compared with the precursor HBPS-2. The molecular weight of the CEPS $\left(M_{\mathrm{n}, \mathrm{GPC}-\mathrm{RI}}=5350 \mathrm{~g} / \mathrm{mol}, \quad M_{\mathrm{n}, \mathrm{GPC} \text { LS }}=14100\right)$ was noticeably higher than the precursor confirming the proposed mechanism.

\section{Conclusion}

In conclusion, a novel approach for the one-pot synthesis of hyperbranched polymers is described. In the method, a special monomer, TEMPOmethacrylate was utilized branching agent as it possesses both vinyl and stable radical moieties in the structure. Thus, a hyperbranched PS with randomly distributed branching points are synthesized in a controlled manner. Moreover, it was also demonstrated that the formed polymer can be used as branched macroinitiator for the chain extension of the polymer chains since the branching TEMPO parts of the polymers can undergo thermally reversible homolysis/recombination reactions.

\section{References}

1. M. Jikei and M.-a. Kakimoto, Prog. Polym. Sci., 26 (2001) 1233.

2. Y. H. Kim, J. Polym. Sci., Part A: Polym. Chem., 36 (1998) 1685.

3. A. Bandyopadhyay, S. Sengupta, and T. Das, "Hyperbranched Polymers for Biomedical Applications", Springer (2017).

4. D. Wang, T. Zhao, X. Zhu, D. Yan, and W. Wang, Chem. Soc. Rev., 44 (2015) 4023.

5. Y. Zheng, S. Li, Z. Weng, and C. Gao, Chem. Soc. Rev., 44 (2015) 4091.

6. X. Zhang, Prog. Org. Coat., 69 (2010) 295.

7. B. Voit, J. Polym. Sci., Part A: Polym. Chem., 43 (2005) 2679.

8. Y. H. Kim and O. W. Webster, Macromolecules, 25 (1992) 5561.

9. J. M. J. Fréchet, M. Henmi, I. Gitsov, S. Aoshima, M. R. Leduc, and R. B. Grubbs, Science, 269 (1995) 1080.

10. C. Aydogan, M. Ciftci, and Y. Yagci, Macromol. Rapid Commun., 39 (2018) 1800276.

11. M. Ciftci, M. U. Kahveci, Y. Yagci, X. Allonas, C. Ley, and H. Tar, Chem. Commun., 48 (2012) 10252.

12. S. Bektas, M. Ciftci, and Y. Yagci, Macromolecules, 46 (2013) 6751.

13. C. Aydogan, M. Ciftci, and Y. Yagci, Macromol.
Rapid Commun., 37 (2016) 650.

14. C. Aydogan, M. Ciftci, V. Kumbaraci, N. Talinli, and Y. Yagci, Macromol. Chem. Phys., 218 (2017) 1700045.

15. E. Murtezi, M. Ciftci, and Y. Yagci, Polym. Int., 64 (2015) 588.

16. S. Balta, C. Aydogan, B. Demir, C. Geyik, M. Ciftci, E. Guler, D. Odaci Demirkol, S. Timur, and Y. Yagci, Macromol. Chem. Phys., 218 (2017) 1700433.

17. T. Isik, M. M. Demir, C. Aydogan, M. Ciftci, and Y. Yagci, J. Polym. Sci., Part A: Polym. Chem., 55 (2017) 1338.

18. C. Geyik, M. Ciftci, B. Demir, B. Guler, A. B. Ozkaya, Z. P. Gumus, F. B. Barlas, D. Odaci Demirkol, H. Coskunol, S. Timur, and Y. Yagci, Polym. Chem., 6 (2015) 5470.

19. M. Seleci, D. A. Seleci, M. Ciftci, D. Odaci Demirkol, F. Stahl, S. Timur, T. Scheper, and Y. Yagci, Langmuir, 31 (2015) 4542.

20. M. Tanabe, G. W. M. Vandermeulen, W. Y. Chan, P. W. Cyr, L. Vanderark, D. A. Rider, and I. Manners, Nat. Mater., 5 (2006) 467.

21. M. Ciftci, G. Yilmaz, and Y. Yagci, J. Photopolym. Sci. Technol., 30 (2017) 385.

22. C. J. Hawker, A. W. Bosman, and E. Harth, Chem. Rev., 101 (2001) 3661.

23. K. Matyjaszewski and J. H. Xia, Chem. Rev., 101 (2001) 2921.

24. J. Chiefari, Y. K. Chong, F. Ercole, J. Krstina, J. Jeffery, T. P. T. Le, R. T. A. Mayadunne, G. F. Meijs, C. L. Moad, G. Moad, E. Rizzardo, and S. H. Thang, Macromolecules, 31 (1998) 5559.

25. J. Nicolas, Y. Guillaneuf, C. Lefay, D. Bertin, D. Gigmes, and B. Charleux, Prog. Polym. Sci., 38 (2013) 63.

26. V. Sciannamea, R. Jerome, and C. Detrembleur, Chem. Rev., 108 (2008) 1104.

27. C. J. Hawker, J. M. J. Frechet, R. B. Grubbs, and J. Dao, J. Am. Chem. Soc., 117 (1995) 10763.

28. Y. F. Tao, J. P. He, Z. M. Wang, J. Y. Pan, H. J. Jiang, S. M. Chen, and Y. L. Yang, Macromolecules, 34 (2001) 4742.

29. A. Z. Niu, C. M. Li, Y. Zhao, J. P. He, Y. L. Yang, and C. Wu, Macromolecules, 34 (2001) 460.

30. S. G. Gaynor, S. Edelman, and K. Matyjaszewski, Macromolecules, 29 (1996) 1079.

31. Z. M. Wang, J. P. He, Y. F. Tao, L. Yang, H. J. Jiang, and Y. L. Yang, Macromolecules, 36 (2003) 7446.

32. C. Aydogan, G. Yilmaz, and Y. Yagci, Macromolecules, 50 (2017) 9115. 